\title{
Acute promyelocytic leukaemia and Hodgkin's disease in the same patient
}

\author{
N G Flanagan, A Whitson, K B Patel, J C Ridway
}

\begin{abstract}
Acute non-lymphocytic leukaemia is a well recognised and increasingly reported complication of treated Hodgkin's disease. The prognosis is generally poor with a disappointing response to chemotherapy. A patient in whom myelodysplastic features appeared after treatment for Hodgkin's disease, to be followed shortly afterwards by acute promyelocytic leukaemia, is reported. Complete remission was achieved and sustained until Hodgkin's disease reappeared three years later when the patient was autografted with a marrow harvested four years earlier. The patient remains in good health with platelet support at the time of writing.
\end{abstract}

(f Clin Pathol 1994;47:565-566)

\section{Case report}

A 28 year old secretary working with British Nuclear Fuels presented in April 1987 with a swelling in the right side of her neck which had been present for four weeks. The swelling had appeared during an upper respiratory tract infection and had initially fluctuated in size. She had no systemic symptoms. In particular, she denied any weight loss, night sweats, or pruritus. She had no clinically important medical history. A blood count taken by her general practitioner had been normal, but the Paul Bunnell test was weakly positive. She was referred to the haematology department with a presumptive diagnosis of atypical infectious mononucleosis.

On examination she looked well and was amply nourished. There was a firm, nontender nodal mass some $8 \mathrm{~cm}$ by $5 \mathrm{~cm}$ in the right anterior triangle, and a smaller mass in the left supraclavicular fossa. No other peripheral adenopathy and no organ enlargement could be detected.

A biopsy specimen of the nodal mass showed nodular sclerosing Hodgkin's disease. A chest radiograph showed very considerable mediastinal disease, confirmed by a computed tomogram. There was also considerable hepatosplenomegaly, with retrocrural, retroperitoneal, and splenic hilar adenopathy. She was classified as clinical stage IVA with liver disease.

Her haematological and biochemical parameters were normal. She was given a standard dose of mustin, vinblastine, procarbazine, prednisolone (MVPP) regimen. In spite of repeated problems of venous access she tolerated six cycles but then declined to continue. A follow up computed tomogram scan confirmed complete remission. In view of the initial bulky chest disease she was given mediastinal radiotherapy, which she completed in mid-1988.

After being symptom free for a few months she began to complain of vague abdominal pain. A computed tomogram in November 1988 showed substantially enlarged nodes at duodenal level. The haematology results, which had become normal after chemotherapy, now showed leucopenia and thrombocytopenia. Marrow examination at this time showed obvious dysplastic changes and raised the possibility that she might be developing leukaemia. A repeat sample three weeks later confirmed acute promyelocytic leukaemia (APML).

She had a greatly increased lactic dehydrogenase value. Cytogenetic analysis revealed a 15:17 translocation. She was treated with a standard protocol of daunorubiun, cytosine arabinoside, C-thioguanine (DAT $3+7$ ) in the hope that this would also have a beneficial effect on her relapsed Hodgkin's disease. She had convincing evidence of complete remission after two courses and all chemotherapy was completed in time for her to go on an overseas holiday in August 1989. A follow up computed tomogram showed no residual Hodgkin's disease. In view of the risk of early relapse, the absence of siblings, and a left ventricular ejection fraction of only $28 \%$, she was booked for an autograft in late 1989. After marrow harvest, she declined to proceed because she was feeling so well.

Her good health continued until late January 1992 when she reported with widespread peripheral adenopathy. A computed tomogram showed multiple deposits in both lung fields with a normal mediastinum. Large node masses were shown in the para-aortic and coeliac areas, the porta hepatis, and in the omentum, liver, and spleen. A marrow sample was normal with normal cytogenetics. Consent was not given for a fine needle biopsy or open biopsy. At this time her alkaline phosphatase activity was greatly increased. In view of the compromised myocardium, she was started on a chemotherapy regimen we would normally reserve for elderly patients with nonHodgkin's lymphoma-CEPP-B (cyclophosphamide, etoposide, procarbazine, bleomycin and prednisolone). She tolerated six monthly courses of this regimen without great

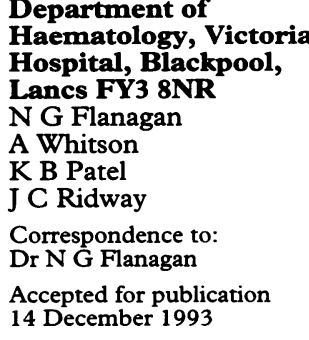


difficulty. She felt very well, there was no palpable disease, and a computed tomogram showed a good partial remission.

At this time the ejection fraction had shown an apparent rise to $35 \%$. She now agreed to autografting and was conditioned with a standard BEAM protocol (BCNU, etoposide, cytosine arabinoside, mephalan). The marrow harvested in 1989 was re-infused without problem in January 1993. She has remained well since. Persistent thrombocytopenia has been a problem, although a follow up marrow sample showed good cellular recovery with apparently ample megakaryocytes.

Occasional red cell support is still needed. The most recent computed tomogram showed no evidence of Hodgkin's disease. Evidence of iron overload has appeared over the past two years with ferritin concentrations of up to $10000 \mu \mathrm{g} / 1$. It was felt that this was a likely contributory factor in her acquiring fairly frequent "nuisance" infections and she was started on regular desferrioxamine with considerable improvement. At the time of writing she is well and active.

\section{Discussion}

This case illustrates several of the complications of Hodgkin's disease, yet progress has been atypical throughout. Much has been written about the risk of leukaemia after treatment for Hodgkin's disease. Brusamolino et $a l^{1}$ found that the risk of treatment related acute non-lymphocytic leukaemia (ANLL) was much greater after combined modality treatment than after either chemotherapy alone or radiotherapy alone, whereas other authors ${ }^{23}$ concluded that the risk was greater after chemotherapy alone than after either of the other regimens. The case reported here is intriguing in that some of the well known features of secondary leukaemia after treatment for Hodgkin's disease are found here although the case is otherwise atypical. For example, she typically displayed myelodysplastic features before developing frank leukaemic changes and she had previously had chemotherapy with radiotherapy. However, the leukaemia proved morphologically and cytogenetically to be APML, an unexpected sequel both to myelodysplasia and treated Hodgkin's disease. The leukaemia appeared only a few months after completing treatment for Hodgkin's disease. The median interval from stopping treatment to leukaemia has been calculated at about 34 months ${ }^{1}$; actuarial analysis suggests that the peak incidence falls between three and nine years after treatment. The short interval in this case raises the possibilities that (a) the leukaemia was part of the natural history of her Hodgkin's disease (cytogenetic studies at the onset of Hodgkin's disease might have been helpful here), and (b) that the appearance of APML following Hodgkin's disease was purely coincidental; or (c) that APML resulted from treatment for Hodgkin's disease, a rarely reported complication. This patient has been remarkably well after her standard treatment for leukaemia and is now in her fourth year of complete remission. The complete remission rate and survival of these patients with secondary ANNL has been disappointingly low, ${ }^{4}$ 5 and prolonged remission is unusual. An approach using marrow transplantation as first line treatment seems more hopeful. ${ }^{67}$ The response to treatment of secondary leukaemia is generally so poor that patients rarely survive long enough to show signs of relapsed Hodgkin's disease. We have found that a rise in the activity of alkaline phosphatase is a warning sign of relapsed Hodgkin's disease: a substantial increase in lactate dehydrogenase is a frequent finding in acute leukaemia. ${ }^{8}$ This case conformed to this with a raised alkaline phosphatase and a normal lactate dehydrogenase activity when the Hodgkin's disease relapsed, and the opposite pattern when APML developed.

We acknowledge the support of Dr P R Kelsey, Professor D Crowther, Dr J Chang and Dr G R Morgenstern.

1 Brusamolino E, Papa G, Valagussa P, Mandelli F, Bernasconi C, et al. Treatment related leukaemia in Bernasconi C, et al. Treatment related leukaemia in
Hodgkin's disease: a multi-institutional study on 75 Hodgkin's disease: a multi-institutic
cases. Haematol Oncol 1987;83:98.

2 Tester WJ, Kinsella TJ, Waller B, et al. Second malignan neoplasms complicating Hodgkin's disease; the National Cancer Institute experience. F Clin Oncol 1984;2:762.

3 Kalder JM, Daws NE, Clarke EA, et al. Leukemia follow ing Hodgkin's disease. N Engl भ Med 1990;322:7-13.

4 Michels SD, McKenna RW, Arthur DC, Brunning RD. Therapy related acute myeloid leukemia and myelodysplastic syndrome. A clinical and morphological study of 65 cases. Blood 1985;65:1364-72.

5 Duane SF, Peterson BA, Bloomfield CD, Michels SA, Hurd DD. Response of therapy associated acute nonHurd DD. Response of therapy associated acute nonMed Paed Oncol 1985;13:207-13.

6 Sargur M, Buckner CD, Appelbaum FR, et al. Marrow transplantation for acute non-lymphocytic Leukaemia following therapy for Hodgkin's disease. $f$ Clin Oncol 1987;5:731.

7 Geller RB, Vogelsang G, Wingard JR. Successful transplantation in acute myelogenous leukaemia followin therapy for Hodgkin's disease. 7 Clin Oncol 1988;6: 1558.

8 Flanagan NG, Ridway JC, Platt CC, Rowlands AJ, Whitsun A. Lactic dehydrogenase estimation in haematological malignancies. Clin Lab Haematol 1989;11: tological 